\section{Differential expression of EFHA2 in cancers of the breast.}
Shahan Mamoor, MS ${ }^{1}$
$3 \quad$ shahanmamoor@gmail.com
East Islip, NY 11730
Breast cancer affects women at relatively high frequency ${ }^{1}$. We mined published microarray datasets ${ }^{2,3}$ to determine in an unbiased fashion and at the systems level genes most differentially expressed in the primary tumors of patients with breast cancer. We report here significant differential expression of the gene encoding EF-hand domain family, member A2, EFHA2, when comparing primary tumors of the breast to the tissue of origin, the normal breast. EFHA2 mRNA was present at significantly lower quantities in tumors of the breast as compared to normal breast tissue. Analysis of human survival data revealed that expression of EFHA2 in primary tumors of the breast was correlated with post-progression survival in patients with basal subtype cancer, demonstrating a relationship between primary tumor expression of a differentially expressed gene and patient survival outcomes influenced by molecular subtype. EFHA2 may be of relevance to initiation, maintenance or progression of cancers of the female breast.

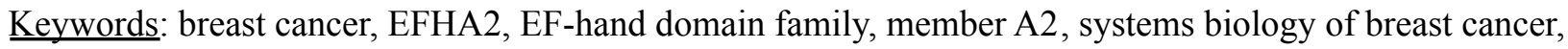
targeted therapeutics in breast cancer. 
Invasive breast cancer is diagnosed in over a quarter of a million women in the United States each year $^{1}$ and in 2018 , breast cancer was the leading cause of cancer death in women worldwide ${ }^{4}$. While patients with localized breast cancer are provided a 99\% 5-year survival rate, patients with regional breast cancer, cancer that has spread to lymph nodes or nearby structures, are provided an $86 \% 5$-year survival rate $^{5,6}$. Patients with metastasis to distant sites, like the brain, are provided a $27 \% 5$-year survival rate ${ }^{5,6}$. Understanding how primary tumors are most transcriptionally different from the tissue from which they originate, the breast, can facilitate development of novel diagnostic and therapeutics to promote early detection and enhanced treatment, and contribute to efforts to prevent progression to metastatic stages. We mined published microarray data ${ }^{2,3}$ to understand at the transcriptome level and in an unbiased fashion genes most differentially expressed in primary tumors of the breast as compared to normal breast tissue. EF-hand domain family, member A2 emerged as among the most differentially expressed genes in cancer of the female breast.

\section{Methods}

We utilized datasets GSE109169² and GSE42568 ${ }^{3}$ for this differential gene expression analysis of female breast cancer. GSE109169 was generated using Affymetrix Human Exon 1.0 ST Array technology with $n=25$ normal breast tissue and $n=25$ tumors of the breast; analysis was performed using platform GPL5175. The tissues from this first dataset are paired tissues ( 25 tumors matching 25 breast tissues from 25 patients). GSE42568 was generated using Affymetrix Human Genome U133 Plus 2.0 array technology with $n=17$ normal breast tissue biopsies and $n=104$ primary breast tumor biopsies from patients with breast cancer; analysis was performed using platform GPL570; the majority of patients whose tumors were analyzed were age 50 or older. The Benjamini and Hochberg method of p-value adjustment was used for ranking of differential expression but raw $p$-values were used to assess statistical significance of global differential expression. Log-transformation of data was auto-detected, and the NCBI generated category of platform annotation was used. A statistical test was performed to evaluate whether EFHA2 expression was significantly different between primary breast tumors and breast tissue using a two-tailed t-test. For Kaplan-Meier survival analysis, we used the Kaplan-Meier plotter online tool ${ }^{7}$ for correlation of EFHA2 mRNA expression levels with post-progression survival (PPS) in $n=36$ patients with basal subtype cancer, $n=76$ patients with basal subtype cancer, $n=38$ patients with luminal B subtype cancer, and $n=30$ patients with HER2+ cancer.

\section{Results}

We performed discovery of genes associated with breast cancer in females by mining two independently published microarray datasets ${ }^{2,3}$.

\section{EFHA2 is differentially expressed in primary tumors of the breast.}

Comparison of 25 normal breast tissues to 25 tumors of the breast ${ }^{2}$ from patients with early-onset breast cancer revealed that the gene encoding the EF-hand domain family, member A2, EFHA2, was among the genes most differentially expressed in tumors of the breast in human breast cancer (Chart 1). When sorting each of the genes expressed in tumors of the breast based on significance of difference as compared to normal breast tissue, EFHA2 ranked 100 out of 19076 total transcripts, equating to $99.5 \%$ differential expression (Chart 1). Differential expression of EFHA2 in female breast cancer was statistically significant (Chart $1 ; p=1.18 \mathrm{E}-14$ ).

Analysis of a second microarray datase ${ }^{3}$, here in 104 tumors from patients aged 31 to 89 at diagnosis, the majority of which were above age 50, again revealed significant differential expression of EFHA2 in human breast cancer (Chart 2). When sorting each of the genes expressed in the tumors of 
patients with breast cancer based on significance of difference as compared to normal breast tissue, EFHA2 ranked 23742 out of 54675 total transcripts, equating to $56.6 \%$ differential expression (Chart 2). Differential expression of EFHA2 in the tumors of patients with breast cancer trended towards statistical significance (Chart $2 ; p=8.56 \mathrm{E}-02$ ). These data suggested that differential expression of EFHA2 was not an artifact of a single microarray dataset, nor was it strictly associated with early-onset breast cancer, rather a general feature of cancers of the breast.

\section{EFHA2 is expressed at significantly lower levels in breast tumors as compared to the breast.}

We obtained exact mRNA expression levels for EFHA2 from the breast and from breast tumors to understand the magnitude and direction of EFHA2 expression change. EFHA2 was expressed at lower levels in tumors of the breast as compared to normal breast tissue (Figure 1). Decreased expression of EFHA2 in primary breast tumors was statistically significant (Figure 1: $p<0.0001$ ). EFHA2 was expressed at $6.74 \pm 0.38$ arbitrary units (AU) in normal breast tissue, while it was expressed at $5.57 \pm 0.40 \mathrm{AU}$ in tumors of the breast. We calculated a mean fold change of 0.83 in EFHA2 mRNA levels when comparing primary tumors of the breast to normal breast tissues.

\section{EFHA2 expression correlates with survival outcomes in basal subtype human breast cancer.}

We performed Kaplan-Meier survival analysis in an attempt to correlate EFHA2 mRNA expression levels with survival outcomes in patients with breast cancer, evaluating correlations between molecular subtype and post-progression survival. We observed a statistically significant correlation between EFHA2 expression and post-progression survival (PPS) in patients with basal subtype breast cancer (Figure 2; $\log$ rank $p$-value: 0.05 for post-progression survival, hazard ratio: 0.44 (0.19-1.02) (Fig. 2)). EFHA2 mRNA levels were a positive prognostic indicator in basal subtype breast cancer patients. Median PPS was 13.35 months for basal patients with low tumor expression of EFHA2 while median PPS was 37.2 months for basal patients with high tumor expression of EFHA2 (Chart 3). EFHA2 primary tumor expression was not correlated with post-progression survival in luminal A subtype (Figure 2; log rank $p$-value: 0.64 for PPS, hazard ratio: 1.15 (0.65-2.04) (Fig. 2), luminal B breast cancer (Figure 2; log rank $p$-value: 0.21 for PPS, hazard ratio: 1.6 (0.77-3.34) (Fig. 2)) or HER2+ breast cancer (Figure 2; log rank $p$-value: 0.77 for PPS, hazard ratio: $1.13(0.49-2.65)$ (Fig. 2)).

Thus, through comparative transcriptome analysis of primary tumors of the breast and normal breast tissue, we found that differential expression and down-regulation of EFHA2 was among the most significant transcriptional features in primary tumors from patients with breast cancer. EFHA2 expression in primary tumors of the breast was correlated with post-progression survival in patients with basal disease, with mRNA levels of EFHA2 a positive prognostic indicator for basal breast cancer patients.

\section{Discussion}

Invasive breast cancer is a medical problem with a $27 \% 5$-year survival rate for women whose disease has spread to distant sites ${ }^{5,6}$. To facilitate understanding of the basic transcriptional differences between primary tumors of the breast and the tissues from which these tumors originate, normal breast tissues, we performed comparative transcriptome analysis using two independently published microarray datasets $^{2,3}$, providing evidence here that differential expression of EF-hand domain family, member A2, encoded by EFHA2, is a defining transcriptional feature of human breast cancer: in early onset-breast cancer, and in patients diagnosed after age 50. EFHA2 was expressed at significantly lower levels in primary tumors from patients with breast cancer as compared to normal breast tissue. Importantly, in patients with basal breast cancer, expression of EFHA2 was correlated with post-progression survival. EF-hand domain family, member A2 and the molecular processes to which it pertains to may be relevant 
to the initiation or progression of human breast cancer.

2

3

4

5

6

7

8

9

10

11

12

13

14

15

16

17

18

19

20

21

22

23

24

25

26

27

28 


\section{References}

1. DeSantis, C.E., Ma, J., Goding Sauer, A., Newman, L.A. and Jemal, A., 2017. Breast cancer statistics, 2017, racial disparity in mortality by state. CA: a cancer journal for clinicians, 67(6), pp.439-448.

2. Chang, J.W., Kuo, W.H., Lin, C.M., Chen, W.L., Chan, S.H., Chiu, M.F., Chang, I.S., Jiang, S.S., Tsai, F.Y., Chen, C.H. and Huang, P.H., 2018. Wild-type p53 upregulates an early onset breast cancer-associated gene GAS7 to suppress metastasis via GAS7-CYFIP1- mediated signaling pathway. Oncogene, 37(30), pp.4137-4150.

3. Clarke, C., Madden, S.F., Doolan, P., Aherne, S.T., Joyce, H., O’driscoll, L., Gallagher, W.M., Hennessy, B.T., Moriarty, M., Crown, J. and Kennedy, S., 2013. Correlating transcriptional networks to breast cancer survival: a large-scale coexpression analysis. Carcinogenesis, 34(10), pp.2300-2308.

4. Bray, F., Ferlay, J., Soerjomataram, I., Siegel, R.L., Torre, L.A. and Jemal, A., 2018. Global cancer statistics 2018: GLOBOCAN estimates of incidence and mortality worldwide for 36 cancers in 185 countries. CA: a cancer journal for clinicians, 68(6), pp.394-424.

5. ACS Cancer Facts \& Figures 2019.

https://www.cancer.net/cancer-types/breast-cancermetastatic/statistics.

6. Survival Rates for Breast Cancer. https://www.cancer.org/cancer/breast-cancer/ understanding-a-breast-cancer-diagnosis/breast-cancer-survival-rates.html

7. Györffy, B., Lanczky, A., Eklund, A.C., Denkert, C., Budczies, J., Li, Q. and Szallasi, Z., 2010. An online survival analysis tool to rapidly assess the effect of 22,277 genes on breast cancer prognosis using microarray data of 1,809 patients. Breast cancer research and treatment, 123(3), pp.725-731. 


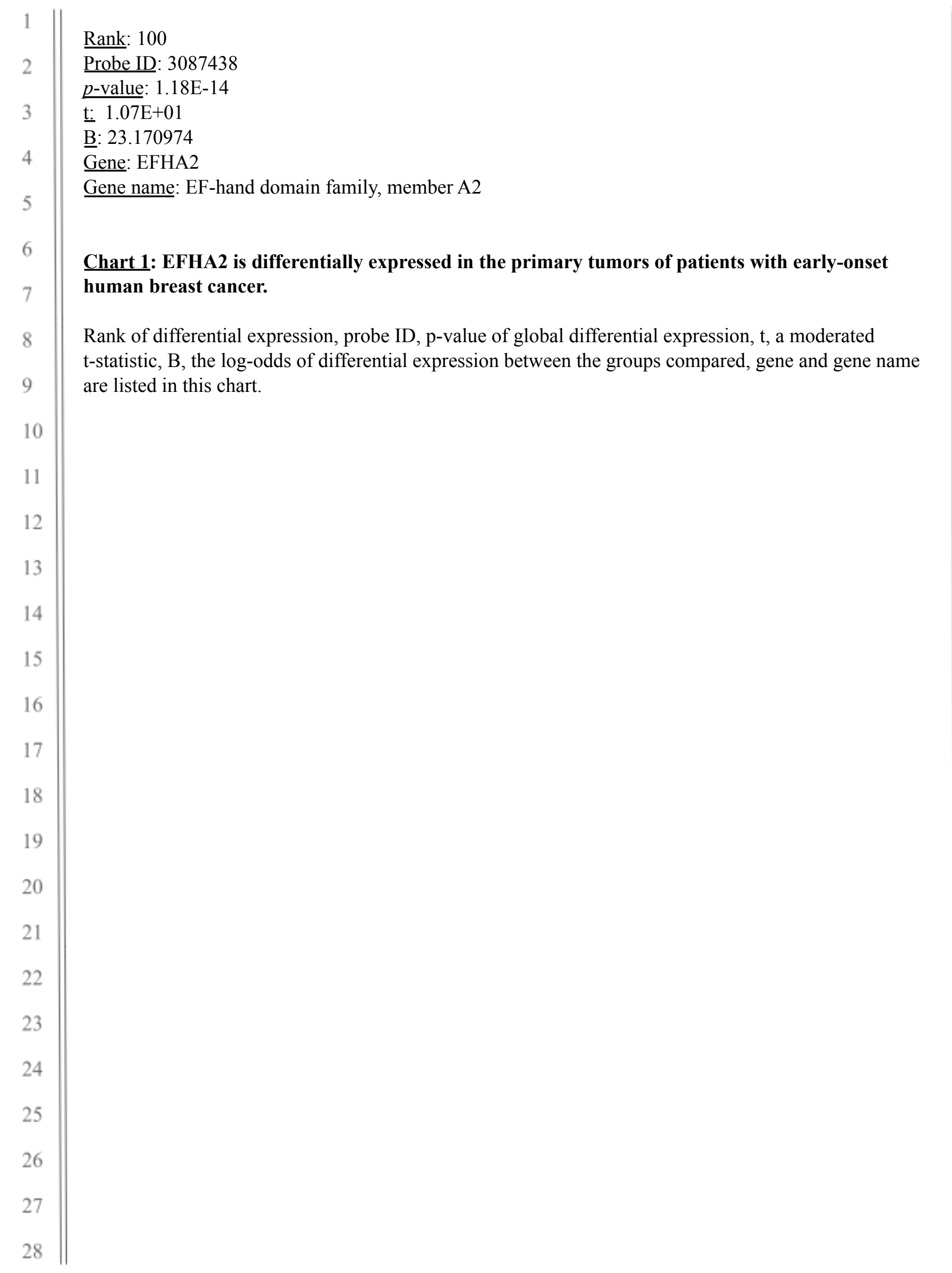




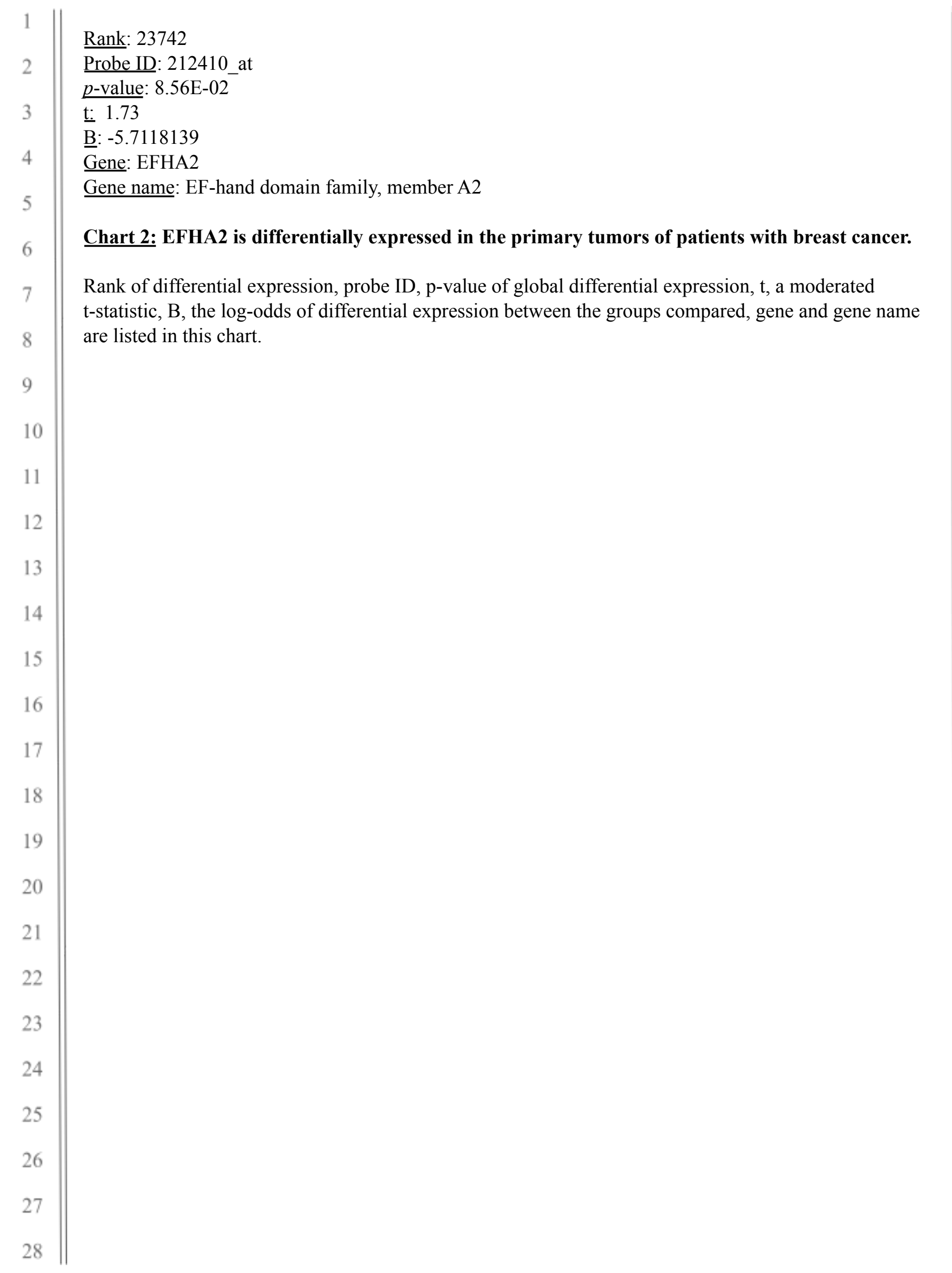




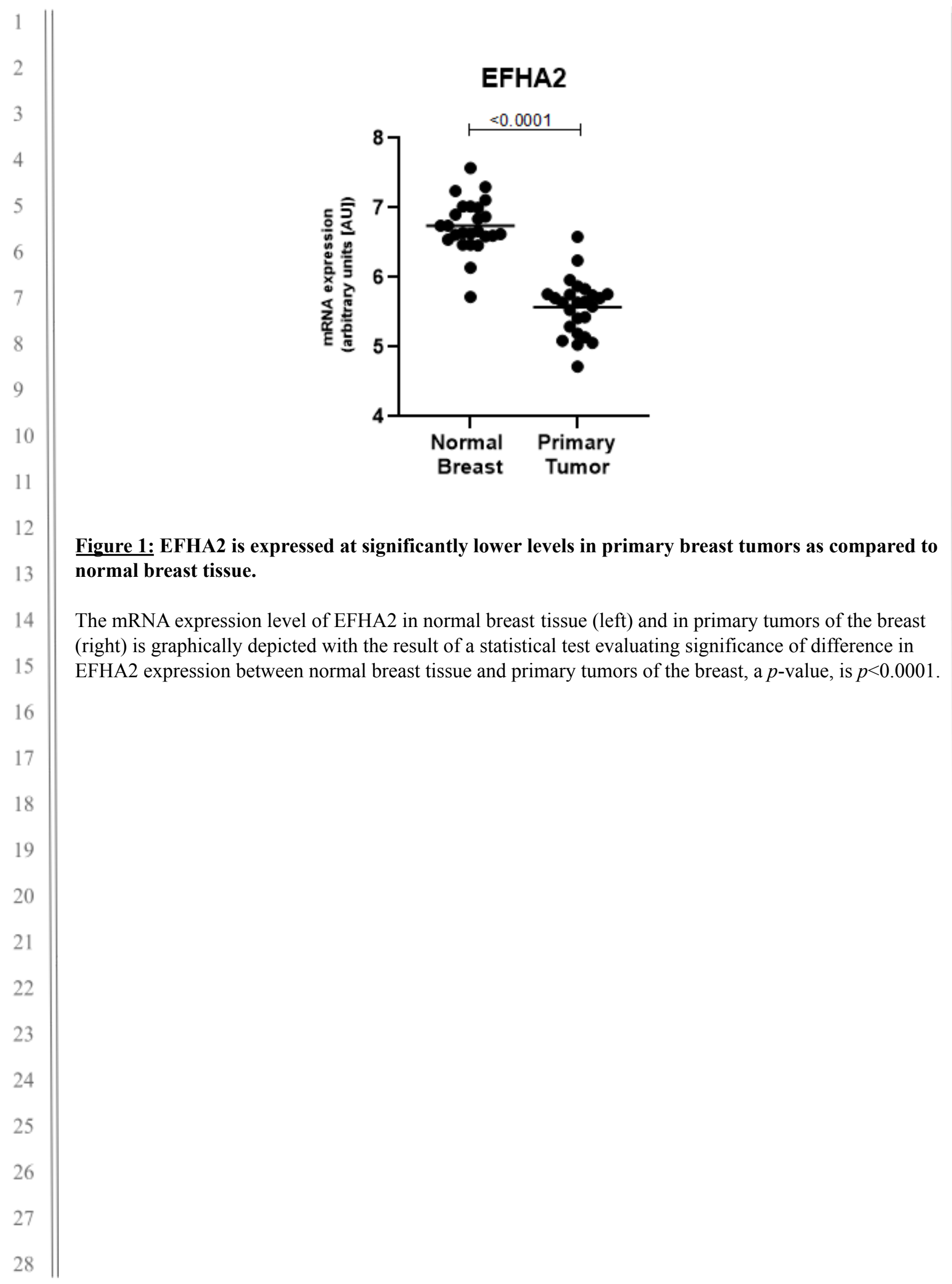




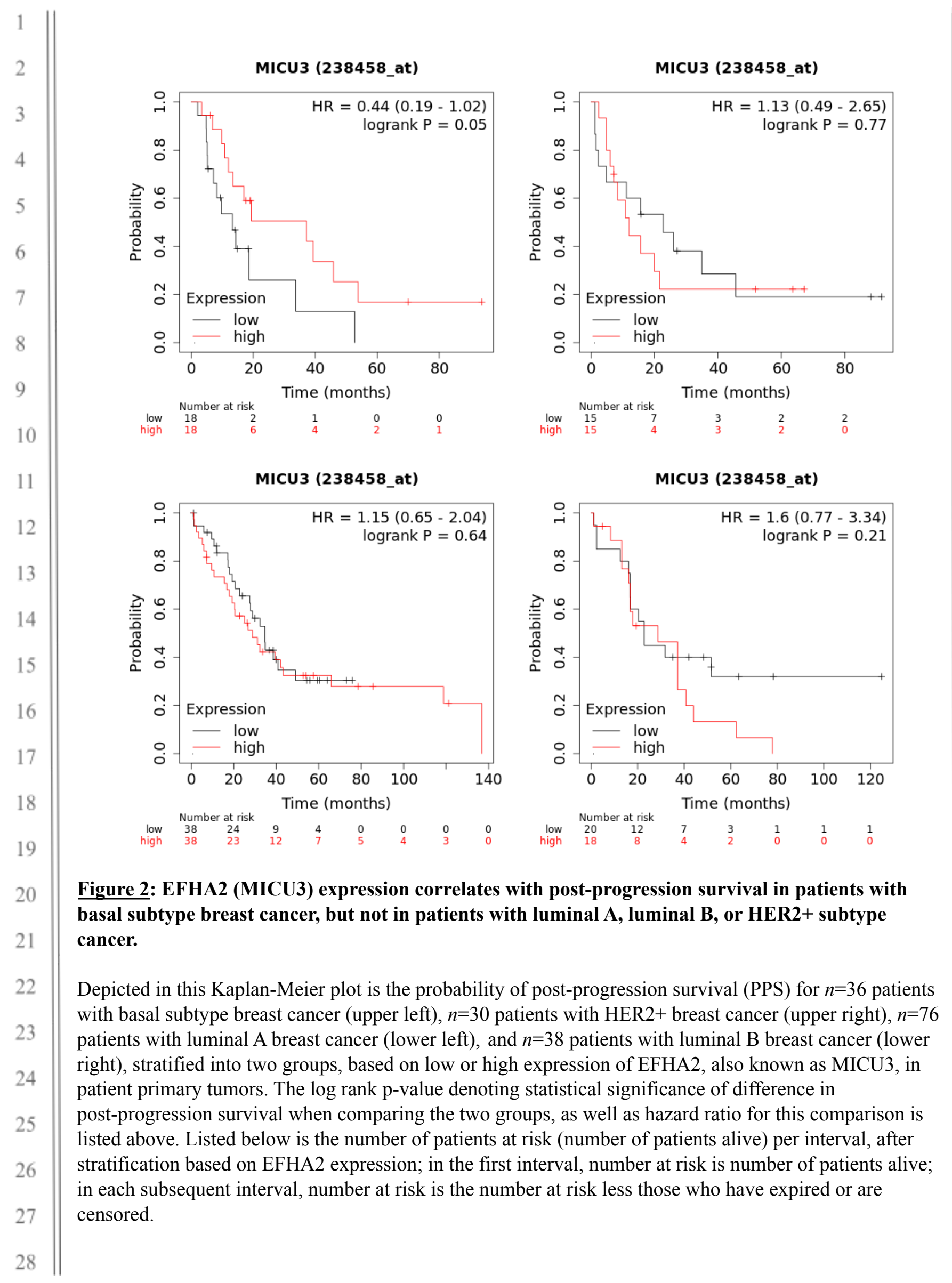




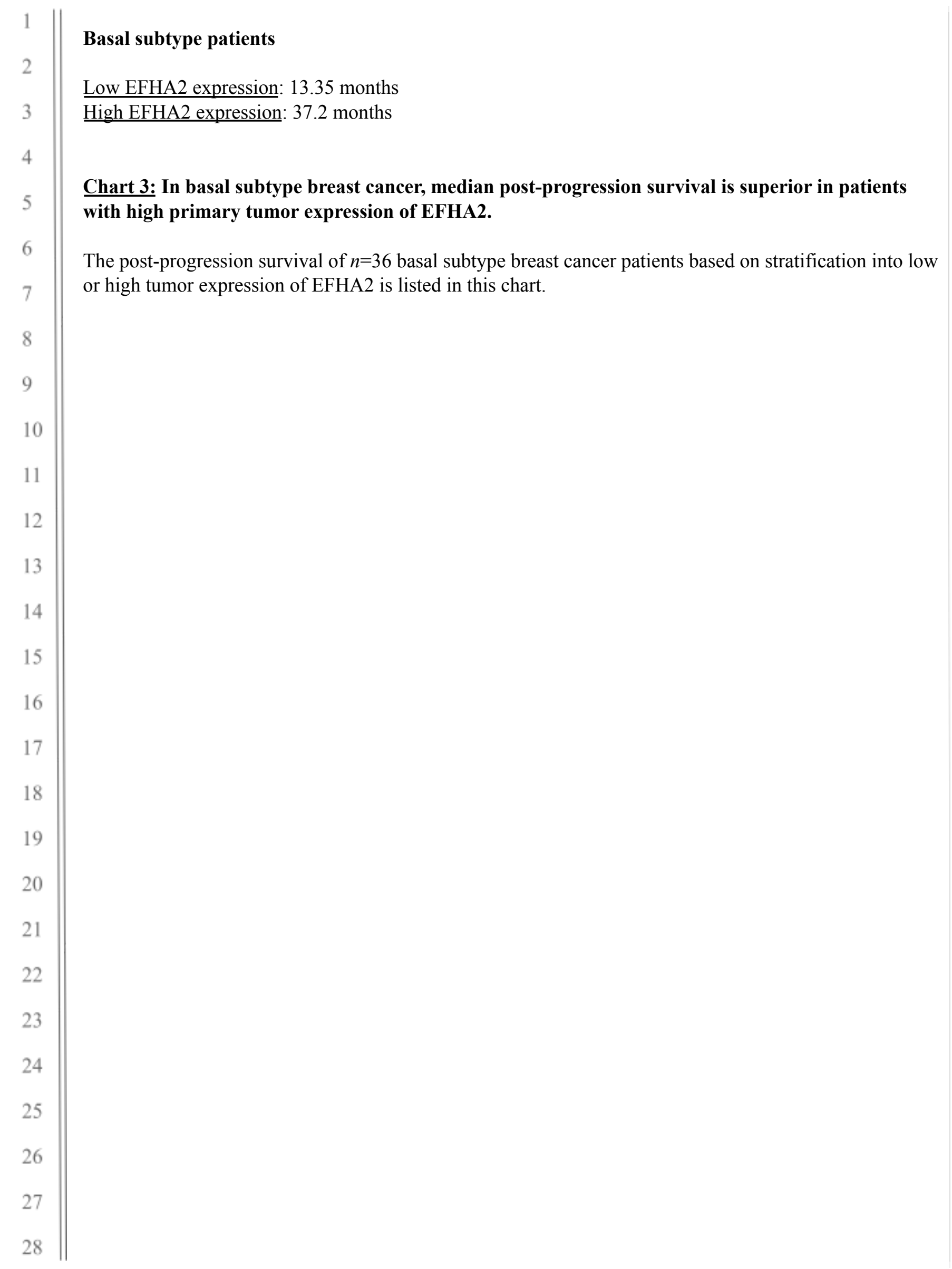

